Research Paper

\title{
Does Lymphadenectomy with at Least 15 Perigastric Lymph Nodes Retrieval Promise an Improved Survival for Gastric Cancer: A Retrospective Cohort Study in Southern China
}

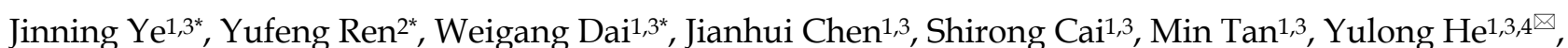
Yujie Yuan ${ }^{1,3}$

1. Center of Gastrointestinal Surgery, The First Affiliated Hospital, Sun Yat-Sen University, Guangzhou, 510080, Guangdong Prov., P.R. China

2. Department of Radiation Oncology, The First Affiliated Hospital, Sun Yat-Sen University, Guangzhou, 510080, Guangdong Prov., P.R. China

3. Center of Gastric cancer, Sun Yat-Sen University, Guangzhou, 510080, Guangdong Prov., P.R. China

4. Center of Digestive Medicine, The Seventh Affiliated Hospital, Sun Yat-sen University, Shenzhen, 518106, Guangdong Prov., P.R. China

*These authors contributed equally to this study.

$\triangle$ Corresponding authors: Dr. Yulong He and Yujie Yuan, Center of Gastrointestinal Surgery, The First Affiliated Hospital, Sun Yat-Sen University, No. 58, 2nd Zhongshan Road, Guangzhou, 510080, Guangdong Province, P.R. China. Tel: +86-20-87755766-8717; Emails: yulongh.sysu@gmail.com (He Y.) and condor.yyj@gmail.com (Yuan Y.).

(C) Ivyspring International Publisher. This is an open access article distributed under the terms of the Creative Commons Attribution (CC BY-NC) license (https://creativecommons.org/licenses/by-nc/4.0/). See http://ivyspring.com/terms for full terms and conditions.

Received: 2018.07.10; Accepted: 2019.01.13; Published: 2019.02.23

\begin{abstract}
Background: Specific guidelines recommend at least 15 or 16 lymph nodes (LNs) be examined to adequately assess nodal category of gastric cancer (GC), but the requirement for minimum number of regional LNs retrieval is not mentioned. This study aims to investigate survival significance from various numbers of perigastric (N1) LNs retrieval and to determine an optimal number harvested in such region.

Study design: From April 1994 to March 2012, 1003 resectable GC patients with at least 15 LNs examined were included. Patients with at least $15 \mathrm{NI}$ nodes retrieval were assigned into study group, with the rest into control group. The 5-year overall survival (OS) rate was compared between two groups, and an optimal number of examined $\mathrm{N} 1$ nodes was detected by a survival joinpoint analysis.

Results: 635 (63.3\%) patients in study group had median 22 (range, 15-75) N1 nodes and 3 (range, 0-74) positive N1 nodes retrieval, with median 10 (range, 0-14) N1 nodes and 1 (range, 0-29) metastatic N1 nodes examined in control group. The number of $\mathrm{N} 1$ nodes retrieval was associated with tumor location $(P=0.007)$, tumor stage $(P<0.001)$ and total number of harvested LNs $(r=0.691, P<0.001)$. Median survival time $(79.0$ vs. 72.0 months, $P=0.462)$ and actual 5 -year $O S$ rate $(41.0 \%$ vs. $39.2 \%, P=0.463)$ were slightly improved in study group compared with control group, with significance obtained via stage-by-stage analysis. The joinpoint analysis indicated that at least seven $\mathrm{N} 1$ nodes retrieval achieved survival significance $(81.0$ vs. 35.0 months, $P=0.036)$, with survival superiority remained until reaching up to $15 \mathrm{~N} 1$ nodes.

Conclusion: Adequate retrieval of perigastric LNs is essential for radical gastrectomy. A harvest of at least 7-15 perigastric LNs could achieve long-term survival benefit for GC patients.
\end{abstract}

Key words: gastric cancer, regional lymph nodes, lymphadenectomy, survival, tumor staging

\section{Introduction}

Lymph node (LN) metastasis is one of the most important determinants of recurrence and long-term survival in patients with gastric cancer (GC). The evaluations of nodal status and extent of LNs retrieval are clinically relevant, since an increased number of involved LNs are the most common mechanism 
leading to upstaging of adenocarcinoma and one of the essential factors in predicting poor prognosis. Up to the present, the proper extent of LNs dissection and retrieval for adequate staging has generated decades of discourse with variable worldwide practice [1,2].

In United States, the National Comprehensive Cancer Network (NCCN) guideline has recommendded radical gastrectomy plus D2 lymphadenectomy as a standard surgical treatment of GC, with an additional goal of removing at least 15 LNs $[3,4]$. However, the optimal number of LNs retrieval for tumor staging is controversial and debatable [5]. Recent studies suggest that tumor staging would be unreliable once less than $10 \mathrm{LNs}$ examined, and the trend towards superior survival can be earned after more than 25 LNs removed [6, 7]. Besides, it is found that the substage-specific survival strongly depends on the harvested number of LNs and culminates in the highest survival at counts of 40 LNs [8]. More evidence has shown that at least 15 LNs retrieval is not sufficient to warrant a recommendation for more curative surgery. Increasing number of regional LNs retrieval, especially around the perigastric region (N1), would be helpful to obtain more examined LNs in theory. Importantly, it is not technically challenging to realize in clinical practice.

To our knowledge, how many LNs should be removed and examined in the first-tier (N1) or perigastric stations is rarely discussed, without valuable recommendations mentioned in available guidelines. Besides, whether the number of perigastric LNs retrieval impacts on tumor staging or prognosis remains unsolved. The current study is designed to explore the role of perigastric LNs retrieval in long-term survival of GC patients and to identify an optimal number for nodal harvest in such region.

\section{Materials and Methods}

This was a retrospective review of prospectively collected GC database at a tertiary-level hospital in Southern China. Between April 1994 and March 2012, adult patients, who had confirmed pathological diagnosis of gastric adenocarcinoma and undergone a radical gastrectomy in our center, were selected from our database. The study protocol was approved by the Institutional Review Board of our hospital, with informed consent waived due to the retrospective study design.

\section{Patients' enrollment}

Within the study period, patients with incomplete or unknown information such as missing tumor stage, number of LNs and follow-up information contained within the dataset were filtered out. Besides, patients with confirmed distant metastasis, including liver, peritoneum, lung, pelvis, bone and brain, were also eliminated. After that, patients with less than $15 \mathrm{LNs}$ isolated were removed from the final analysis. All data on age, gender, body mass index (BMI), tumor location, surgical procedure, type of lymphadenectomy, harvested LNs, positive LNs and long-term survival were taken directly from original dataset, with no need for additional recoding or calculations. As described by others, tumor features, such as depth of invasion, histology, LNs metastases and pathological staging, were determined on basis of the $7^{\text {th }}$ edition of American Joint Committee on Cancer (AJCC) staging system for GC [9]. Type of radical surgery was divided into total gastrectomy and subtotal gastrectomy mainly based on the principle of " $r x$ sum-surg prim site (1998+)". If a definitive surgical procedure was missing or beyond the above-mentioned scope, the patient would be filtered from the study as that not undergoing radical gastrectomy. Besides, the following groups of patients were also eliminated: multiple primary tumors existed, <3-month survival and follow-up period.

\section{Clinical management schemes}

All patients were allocated a clinical tumor stage (cTNM) during the multidisciplinary team (MDT) meeting, which was usually held once a week. Routinely, all surgical procedures and systemic chemotherapies were determined by the MDT discussion. The detailed surgical approach was primarily dictated by surgeon's preference, with open or laparoscopic fashion performed across the period. However, D2 or D2 plus lymphadenectomy were performed by highly experienced gastrointestinal surgeons, as described previously [10]. Specifically, LN harvest was performed by one of scrubbing surgeons in the theater, with various stations of removed LNs identified prior to pathologic assessment. Thereafter, the definitive numbers of harvested and metastatic nodes were confirmed microscopically by experienced pathologists. As for chemotherapy, fluorouracil (5-Fu)-based chemotherapy was performed for patients with stage II or higher, with combining S-1 $\left(60 \mathrm{mg} / \mathrm{m}^{2}\right)$ and oxaliplatin $\left(85 \mathrm{mg} / \mathrm{m}^{2}\right)$ as a main regimen for at least eight cycles in planning. Besides, adjuvant radiotherapy would be performed in certain patients with regional LN metastasis or suspicious positive surgical margin from final pathological report. Neoadjuvant therapy mainly performed as chemotherapy was suggested to patients with unresectable tumor or severe LN metastasis.

Follow-up investigations were typically scheduled every three months for the first two years after surgery, every six months for the next three years and then once a year until the patient's death [11]. The 
follow-up program included a physical examination, physical and psychological assessments, laboratory blood tests, digestive tumor markers monitor and contrast-enhanced computed tomography (CT). Recurrence was confirmed on the basis of clinical, radiologic or endoscopic signs of disease.

\section{Study design and outcomes}

According to the definition of LN stations by the Japanese Research Society for GC study, LN stations 1-6 are referred to as N1, stations 7-11 as N2, stations $12-14$ as N3, and stations 15 to 16 as N4 [12]. Hence, D2 lymphadenectomy should include perigastric LNs with additional nodes along left gastric, common hepatic, celiac, splenic arteries or splenic hilum. In this study, patients with qualified number of LNs retrieval ( $\geq 15$ nodes) were enrolled and stratified into two groups: control group with less than $15 \mathrm{~N} 1$ nodes and study group with at least $15 \mathrm{~N} 1$ nodes.

The primary outcome was the 5-year overall survival (OS) after radical gastrectomy. The secondary outcomes, including length of stay, postoperative complications and 5-year disease-free survival (DFS), were also explored in such cohort. Comparisons between study group and control group were further performed using subgroup analysis of tumor stage. In addition, the optimum number of harvested LNs in N1 stations for prognostic significance was calculated by a joinpoint trend analysis.

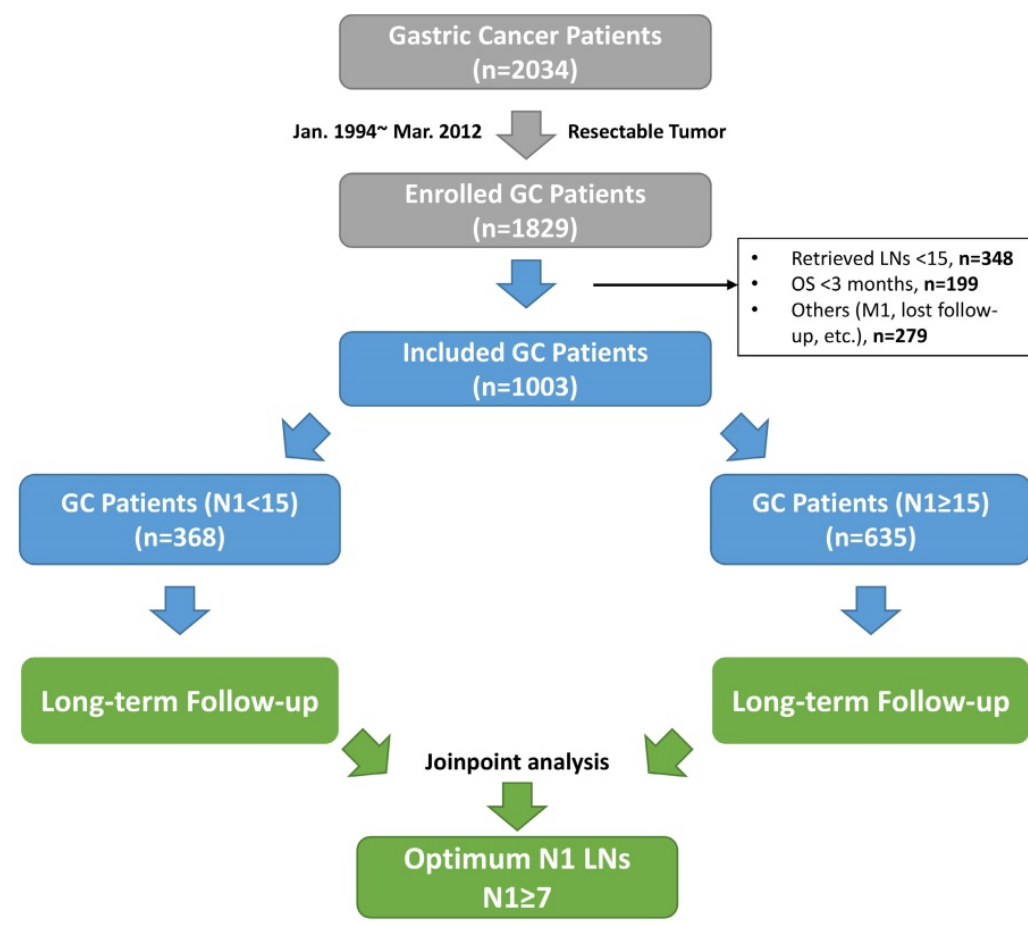

Figure 1. The flow chart of this study. Abbreviations: GC, gastric cancer; LNs, lymph nodes; OS, overall survival; $\mathrm{Ml}$, synchronized tumor isolated outside of stomach; $\mathrm{N} 1$ stations, the first-tier lymph node stations (No. 1-6 stations).
The OS time was calculated from the date of surgery to death from any cause. The DFS time was calculated from the date of surgery to confirmed recurrence, occurrence of a new primary cancer or cancer-related death. The last follow-up date was December 31, 2017.

\section{Statistical analysis}

Descriptive statistics were employed to present demographic characteristics and oncologic outcomes. Data were expressed as mean \pm standard deviation (SD) if not indicated otherwise. Survival analysis was performed using the Kaplan-Meier method and compared with the log-rank test. Student $t$ test and Mann-Whitney $U$ test were employed for continuous variables, whereas Fisher's exact test and $\chi^{2}$ test were used for categorical variables. Joinpoint trend analysis by using median survival at various numbers of LNs retrieved in N1 stations as a dependent variable was performed to identify an optimal number of N1 nodes. A multivariate analysis with cox-regression method was appended to explore risk factors affecting long-term outcomes. All analyses were performed using IBM ${ }^{\circledR}$ SPSS $^{\circledR}$ Statistics (Ver. 23.0; Chicago, IL). Two-tailed tests were used, with $P$ values $<0.05$ considered statistically significant.

\section{Results}

Within the study period, 2034 GC patients were first selected from our database. Among those, 205 patients with unresectable tumor were removed, with additional 826 patients excluded based on the exclusion criteria. In sum, 1003 GC patients were included for the final analysis. The flowchart of patient selection and study design is shown in Figure 1. Of those included, the median age was 58 (range, 14-85) years, with a majority of male patients joined $(66.1 \%)$. The median follow-up period of this cohort was 47 (range, 15-150) months, with 9\% of missing follow-up data recorded. Specifically, $635(63.3 \%)$ patients were assigned to study group, with the rest into control group. The observed mortality rate of this cohort was $16.0 \%$ and $46.4 \%$ at 1 and 5 years, respectively.

Demographics and tumor features of included subjects are presented in Table 1. General information and baseline characteristics, such as gender, BMI, comorbidity, histology and surgical procedure, were comparable between both groups $(P>0.05)$. However, tumor 
location $(P=0.007)$, number of retrieved and positive LNs $(P<0.001)$, and pathological stage $(P<0.001)$ were significantly different between two groups.

\section{Lymph nodes dissection and retrieval}

In the current cohort, $95.3 \%$ of subjects received at least D2 lymphadenectomy during radical gastrectomy, with only 47 (4.7\%) patients undergoing D1 lymphadenectomy due to early tumor stage. The median number of harvested LNs was 29 (range, 15-150), with median value of 3 (range, 0-82) for positive LNs. The frequency distributions of LNs retrieval across entire cohort are summarized in Figure 2. Additionally, the median number of N1 nodes retrieval for all patients was 17 (range, 0-75), with median value of 2 (range, 0-74) for N1 nodal metastasis. The number of retrieved N1 nodes in study group was twice more than control group [median, 22 (range, 15-75) vs. 10 (range, 0-14); $P<0.001]$, with similar finding for positive N1 nodes [median, 3 (range, 0-74) vs. 1 (range, 0-29); $P<0.001$ ]. However, the number of isolated LNs in N2 stations was identical in both groups [median, 8 (range, 0-49) vs 8 (range, $0-32$ ); $P=0.077]$. Of note, 353 (35.2\%) patients underwent curative surgery 10 years ago, with the rest receiving such operation in recent 10 years. The comparisons between those in two different time periods showed that the number of LNs retrieval was significantly increased in total (mean, $30.7 \pm 14.8$ vs. $33.1 \pm 13.8 ; P=0.012$ ) and $\mathrm{N} 2$ stations (mean, $7.9 \pm 5.9$ vs. $10.3 \pm 7.5 ; P<0.001$ ), but a little improved in N1 stations (mean, $19.6 \pm 11.1$ vs. 18.6 \pm 9.7 ; $P=0.133$ ). However, the number of positive LNs retrieval was not significantly different between before and after the decade.

A correlated analysis indicated that total gastrectomy was associated with more LNs harvested in total $(P=0.032)$ and N1 stations $(P=0.025)$ both than subtotal gastrectomy along with D2 lymphadenectomy. More importantly, additional analyses suggested that total harvested number of LNs was strongly associated with isolated number of positive LNs and perigastric LNs, respectively (Figure 3). Meanwhile, increasing retrieval of N1 nodes was also associated with an increased number of metastatic nodes in such region $(r=0.434, P=0.463)$.

\section{Primary and secondary outcomes}

Laparoscopic surgery was performed in 126 $(12.6 \%)$ patients, with pT1 stage confirmed in most of cases. Intraoperative conversion from laparoscopic to open gastrectomy was not observed. The median length of stay after surgery was 13 (range, 7-117) days. The in-hospital mortality could not be evaluated due to the exclusion criteria. Within 30 days of postoperative observation, $235(23.4 \%)$ patients developed various degrees of complications, with detailed results summarized in Table 2 . Briefly, the incidence of postoperative complications was relatively increased in study group compared with control group, with no significant difference found $(24.7 \%$ vs. $21.2 \%, P=0.216)$. Besides, the rate of intraoperative transfusion was slightly increased $(39.7 \%$ vs. $36.1 \%, P=0.593)$, similarly as for the operative time $(P=0.693)$.

Table 1. Demographic and baseline characteristics of patients with gastric cancer

\begin{tabular}{|c|c|c|c|c|}
\hline Parameter & $\begin{array}{l}\text { The } \\
\text { Pooled } \\
\left(\sum n \geq 15\right)\end{array}$ & $\begin{array}{l}\text { Study } \\
\text { group } \\
\left(\sum N 1 \geq 15\right)\end{array}$ & $\begin{array}{l}\text { Control } \\
\text { group } \\
\left(\sum N 1<15\right)\end{array}$ & P value \\
\hline & $(n=1003)$ & $(\mathrm{n}=635)$ & $(n=368)$ & \\
\hline Age, yrs & $56.6 \pm 12.3$ & $56.6 \pm 12.0$ & $56.6 \pm 12.8$ & 0.931 \\
\hline$\leq 65$ & $741(73.9)$ & $475(74.8)$ & $266(72.3)$ & 0.421 \\
\hline$>65$ & $262(26.1)$ & $160(25.2)$ & $102(27.7)$ & \\
\hline Gender, Male:Female & $663: 340$ & $422: 213$ & 241:127 & 0.782 \\
\hline BMI, kg/m2 & $21.6 \pm 3.3$ & $21.5 \pm 3.4$ & $21.7 \pm 3.1$ & 0.638 \\
\hline \multicolumn{5}{|l|}{ Comorbidity (yes), n (\%) } \\
\hline HTN & $260(24.8)$ & $152(23.9)$ & $98(26.6)$ & 0.364 \\
\hline DM & $115(11.5)$ & $65(10.2)$ & $50(13.6)$ & 0.123 \\
\hline Smoke (yes), n (\%) & $283(28.2)$ & $183(28.8)$ & $100(27.1)$ & 0.611 \\
\hline Alcohol abuse (yes), n (\%) & $190(18.9)$ & $123(19.4)$ & $67(18.2)$ & 0.677 \\
\hline ASA, n (\%) & & & & 0.325 \\
\hline I+II & $754(75.2)$ & $484(76.2)$ & $270(73.4)$ & \\
\hline III+IV & $249(24.8)$ & $151(23.8)$ & $98(26.6)$ & \\
\hline Albumin level, g/dL & $38.8 \pm 6.4$ & $38.5 \pm 6.9$ & $39.3 \pm 5.5$ & 0.057 \\
\hline Tumor Location, n (\%) & & & & 0.007 \\
\hline Upper $1 / 3$ & $347(34.6)$ & $225(35.4)$ & $122(33.1)$ & \\
\hline Middle $1 / 3$ & $279(27.8)$ & $193(30.4)$ & $86(23.4)$ & \\
\hline Lower $1 / 3$ & $377(37.6)$ & $217(34.2)$ & $160(43.5)$ & \\
\hline Type of Anastomosis, n (\%) & & & & 0.629 \\
\hline Billroth I & $34(3.4)$ & $23(3.6)$ & $11(3.0)$ & \\
\hline Billroth II & $340(33.9)$ & $209(32.9)$ & $131(35.6)$ & \\
\hline Roux-en-Y & $629(62.7)$ & $403(63.5)$ & $226(61.4)$ & \\
\hline Lymphadenectomy, n (\%) & & & & 0.800 \\
\hline D1 & $47(4.7)$ & $28(4.4)$ & $19(5.2)$ & \\
\hline D2 & $784(78.2)$ & $500(78.8)$ & $284(77.1)$ & \\
\hline D2 plus* & $172(17.1)$ & $107(16.8)$ & $65(17.7)$ & \\
\hline Depth of invasion, $\mathrm{n}(\%)$ & & & & 0.377 \\
\hline pT0-1 & $157(15.6)$ & $97(15.3)$ & $60(16.3)$ & \\
\hline pT2 & $103(10.3)$ & $58(9.1)$ & $45(12.2)$ & \\
\hline pT3 & $553(55.1)$ & $360(56.7)$ & $193(52.5)$ & \\
\hline pT4 & $190(19.0)$ & $120(18.9)$ & $70(19.0)$ & \\
\hline Number of LNs retrieval, $\mathrm{n}$ & $32.2 \pm 14.2$ & $37.2 \pm 14.7$ & $23.6 \pm 8.0$ & $<0.001$ \\
\hline N1 stations & $18.9 \pm 10.2$ & $24.3 \pm 8.7$ & $9.5 \pm 3.6$ & $<0.001$ \\
\hline N2 stations & $9.4 \pm 7.0$ & $9.7 \pm 7.6$ & $8.9 \pm 5.9$ & 0.077 \\
\hline N2 plus stations & $1.7 \pm 3.9$ & $1.8 \pm 4.3$ & $1.6 \pm 3.1$ & 0.352 \\
\hline Number of Positive LNs, $\mathrm{n}$ & $7.3 \pm 11.1$ & $8.9 \pm 12.8$ & $4.7 \pm 6.3$ & $<0.001$ \\
\hline Histopathological type, n (\%) & & & & 0.149 \\
\hline Well diff. & $81(8.1)$ & $55(8.7)$ & $26(7.1)$ & \\
\hline Moderate diff. & $347(34.6)$ & $206(32.4)$ & $141(38.3)$ & \\
\hline Poor diff. & $575(57.3)$ & $374(58.9)$ & $201(54.6)$ & \\
\hline pTNM Stage, n (\%) & & & & $<0.001$ \\
\hline I & $187(18.6)$ & $100(15.7)$ & $87(23.6)$ & \\
\hline II & $425(42.4)$ & $263(41.4)$ & $162(44.0)$ & \\
\hline III & $391(39.0)$ & $272(42.9)$ & $119(32.4)$ & \\
\hline
\end{tabular}

Patients were divided into study group and control group according to the number of N1 station retrieval (cutoff value, 15). Data present with mean \pm SD or number (percentage of column). Abbreviations: BMI, body mass index; HTN, hypertension; DM, diabetes mellitus; ASA, American society of anesthesia; diff., differentiation. * D2 plus means extended lymphadenectomy beyond D2 stations during radical gastrectomy. 
Table 2. The short-term outcomes after a definitive operation for gastric cancer

\begin{tabular}{lllll}
\hline Parameter & $\begin{array}{l}\text { The Pooled } \\
\text { (n=1003) }\end{array}$ & $\begin{array}{l}\text { Study } \\
\text { group } \\
\text { (n=635) }\end{array}$ & $\begin{array}{l}\text { Control } \\
\text { group } \\
\text { (n=368) }\end{array}$ & P value \\
\hline Intraoper. transfusion, n (\%) & & & 0.593 \\
None & $618(61.6)$ & $383(60.3)$ & $235(63.9)$ & \\
$<500 \mathrm{~mL}$ & $177(17.6)$ & $116(18.3)$ & $61(16.6)$ & \\
$500-1000 \mathrm{~mL}$ & $166(16.6)$ & $107(16.9)$ & $59(16.0)$ & \\
$>1000 \mathrm{~mL}$ & $42(4.2)$ & $29(4.6)$ & $13(3.5)$ & \\
Operative time, min & $282 \pm 77$ & $283 \pm 78$ & $281 \pm 76$ & 0.693 \\
LOS, day & $25.0 \pm 10.1$ & $25.1 \pm 10.5$ & $24.6 \pm 9.5$ & 0.486 \\
LOPS, day & $15.0 \pm 8.7$ & $15.1 \pm 9.2$ & $14.7 \pm 7.8$ & 0.493 \\
Postoper. complications & $235(23.4)$ & $157(24.7)$ & $78(21.2)$ & 0.216 \\
(yes), n (\%) & & & & \\
Pyrexia & $105(10.5)$ & $72(11.3)$ & $33(9.0)$ & \\
SSI & $58(5.8)$ & $38(6.0)$ & $20(5.4)$ & \\
Bowel obstruction/ileus & $34(3.4)$ & $22(3.5)$ & $12(3.3)$ & \\
Anastomotic leak & $25(2.5)$ & $16(2.5)$ & $9(2.4)$ & \\
IAH & $17(1.7)$ & $11(1.7)$ & $6(1.6)$ & \\
Others & $25(2.5)$ & $16(2.5)$ & $9(2.4)$ & \\
\hline
\end{tabular}

Abbreviations: Intraoper., intraoperative; Postoper., postoperative; LOS, length of stay; LOPS, length of postoperative stay; SSI, surgical site infection; IAH, intra-abdominal hemorrhage. Others include several rare complications, such as dumping syndrome, digestive fluid reflux, persistent vomiting and diarrhea.
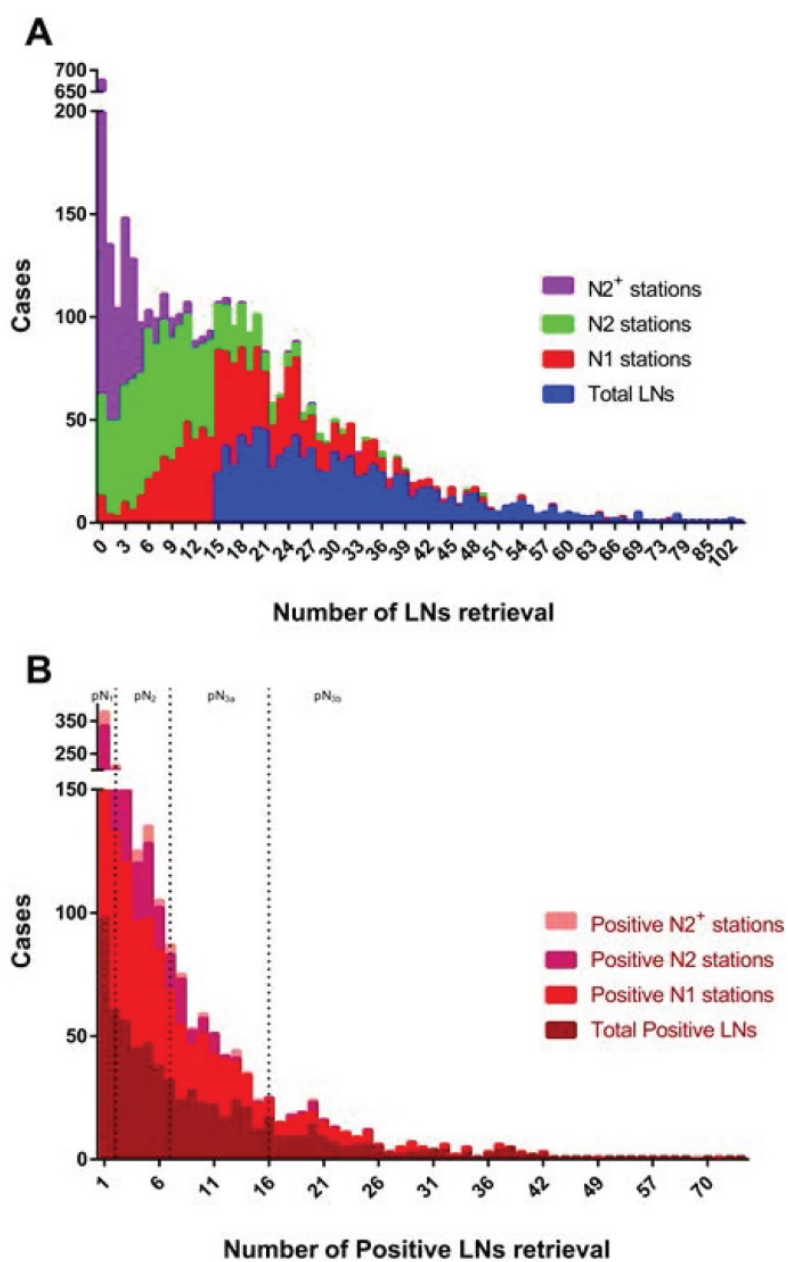

Figure 2. The frequency distributions of lymph nodes retrieval. Cases with less than 15 LNs retrieved in total were excluded from this study. Right-skewed distribution is observed in either number of $L N s$ retrieval $(A)$ or positive LNs retrieval (B). N2 stations include No. 7-12 stations, with N2+ stations including No. 13 station and above stations. pN1 stage presents with $1 \sim 2$ positive $L N s$ retrieved in all stations, with $\mathrm{pN} 2$ for $3 \sim 7$ positive $L N s$, $p N 3 a$ for $8 \sim 16$ positive LNs and pN3b for at least 17 positive LNs.
Neoadjuvant chemotherapy was not recorded in this cohort. Adjuvant chemotherapy, mainly applied with SOX regimen, was selectively performed in 641 $(63.9 \%)$ patients with pathological stage IIB and above. No severe adverse event associated with chemotherapy was observed. Within available followup period, the OS rate was $38.5 \%$, as all stages taken together. The median survival time across the entire cohort was 79.0 (95\% CI, 60.0-98.0) months, calculated as 79.0 months for the study group and 72.0 months for the control group. The actual 5-year OS rate was similar between two groups for all stages $(41.0 \%$ vs. $39.2 \%, P=0.463)$. However, by further stage-by-stage analysis, the actual 5-year OS rates for stage IB $(94.0 \%$ vs. $85.4 \%, P=0.021)$, IIA ( $70.8 \%$ vs. $76.5 \%, P=0.026)$, IIB (61.6\% vs. $50.8 \%, P=0.007)$, IIIA $(36.5 \%$ vs. $21.5 \%$, $P=0.019)$ and IIIC $(19.3 \%$ vs. $10.9 \%, P=0.007)$ were significantly different between groups (Figure 4). The findings suggested that patients with at least $15 \mathrm{LNs}$ harvested in N1 station almost had a better long-term survival, with except for those with stage IA and IIA.

Similar finding was observed for the actual 5 -year DFS. Briefly, the overall recurrence rate was $5.5 \%(55 / 1003)$ within the first five years of follow-up period, with no recurrence occurred in patients with stage IA or IB. The actual 5 -year DFS rate was $84.4 \%$ in the study group and $65.6 \%$ in the control group for patients with stage IIA, $77.4 \%$ and $62.3 \%$ for stage IIB, $59.5 \%$ and $52.7 \%$ for stage IIIA, $51.2 \%$ and $46.4 \%$ for stage IIIB, and $32.6 \%$ and $29.5 \%$ for stage IIIC, respectively. A comparative analysis of the DFS indicated survival significance only emerged in stage II cancer.

\section{Joinpoint and multivariate analyses}

A joinpoint trend analysis of median survival found two major joinpoints to maintain survival benefit: 7 and $15 \mathrm{~N} 1$ nodes, respectively (Figure 5). Specifically, the joinpoint model of overall survival became insignificant after the first joinpoint, which indicated that at least $7 \mathrm{~N} 1$ nodes retrieval would achieve an optimal long-term survival $(P=0.036)$. After that, similar median survival time was obtained along with increasing amount of $\mathrm{N} 1$ nodes until reaching up to 15 nodes. Corresponding KaplanMeier analysis by using stage-by-stage comparison demonstrated an overall survival significance under at least $7 \mathrm{~N} 1$ nodes examined $(P=0.003)$. At last, a multivariate analysis, which employed gender, age (>65 years), BMI $\left(>18.0 \mathrm{~kg} / \mathrm{m}^{2}\right)$, tumor location, tumor stage (>IIA), pathologic grade, and amount of N1 nodes retrieval $(\geq 15)$ to predict survival benefit, indicated that the male $(P=0.016)$, advanced stage $(P=0.028)$ and less than $15 \mathrm{~N} 1$ nodes retrieval $(P=$ 0.042 ) were independent risk factors of poor survival. 


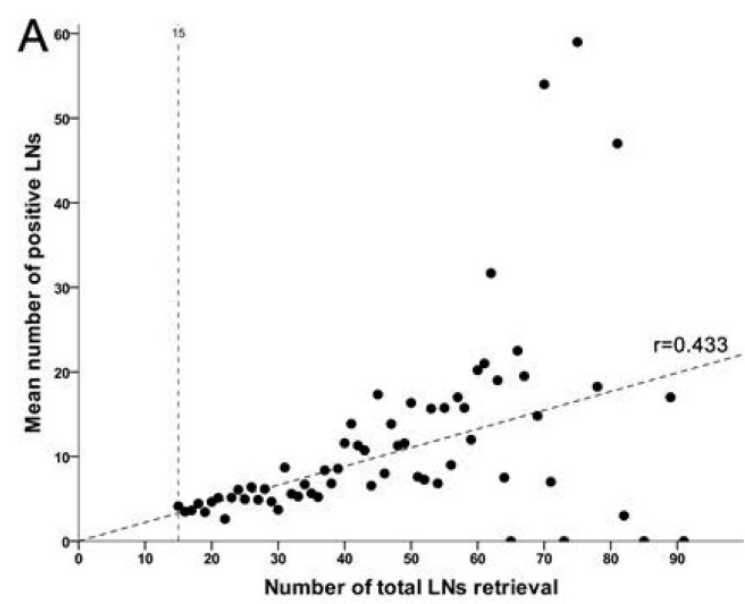

time-period of surgery. More importantly, the findings confirmed that lymphadenectomy with at least 15 perigastric nodes retrieval could earn improved survival, especially for stage IIB and above cancer. Finally, a joinpoint trend analysis indicated an optimal count of 7 LNs retrieval in N1 stations would achieve long-term survival significance in the current cohort.

The novelties of this study were threefold. First, a specific investigation of survival benefits for increasing number of perigastric LNs retrieval was performed with a large sample size and strict selection criteria. All cases with at least 15 harvested LNs by our surgeons could be qualified to enroll for the survival analysis. Second, the association between perigastric LNs dissection and pathological tumor stage, especially for nodal category, was explored with several correlation analyses. Our results indicated that the quantity of perigastric LNs retrieval could directly reflect metastatic number of LNs in N1 stations and total number of LNs in all regions. The confirmed relationship indicated that using perigastric LNs amount to simply evaluate the prognosis of GC would be practicable and reasonable in advance of available pathological staging. Third, a joinpoint trend analysis was successfully applied based on the large sample size, and provided two optimal cut-off values for increasing perigastric LNs retrieval to achieve survival significance for GC patients. The findings from such trend analysis indirectly suggested a rational value on the harvest of perigastric LNs when dissecting nodes from the specimen.

LN metastasis is one of the strongest predicators of poor prognosis in GC, with the anatomic and numerical extent of nodal dissection prognostic of oncologic outcomes [13]. Nodal-positive GC usually indicates a regional spread of cancer cells and increased risk of recurrence, as compared to nodal-negative disease. In most western GC centers, LNs are retrieved by experienced pathologists after surgery [14], whereas in our center, LNs are dissected by qualified surgical trainees in the theater within 30 minutes once the specimen isolated from the abdomen. It has shown that an immediate LN retrieval by surgeons is associated with markedly increased number of both total and perigastric LNs compared with that by pathologists [15].

In the last two decades, numerous studies have

\section{Discussion}

In this study, the results suggested that the harvested number of perigastric LNs was positively correlated with tumor location and tumor stage, but not related to extension of lymphadenectomy or confirmed the role of adequate number and dissection regions of LNs retrieval in proper $\mathrm{N}$ staging and planning appropriate GC treatment regimens [11, 16-20]. Generally, incremental number of LNs retrieval is directly correlated with improved survival, and inadequate lymphadenectomy or nodal 
retrieval is implicated in understaging of nodal disease [21]. Recently, a large international retrospective study, which combined the Surveillance, Epidemiology, and End Results program database and a single-center database for long-term survival evaluation, has shown that the maximal survival advantage could be obtained by performing the lymphadenectomy with at least 29 removed LNs [13].
In clinical practice, 29 nodes retrieval is not challenging, since the reported average amount of harvested LNs has ranged from 33 to 47 by many well-conducted trials of radical gastrectomy in China, Italy, Japan and South Korea [22-26]. Unfortunately, the minimal requirement for harvested number of perigastric LNs is rarely reported, with no guideline mentioned such requirement yet.
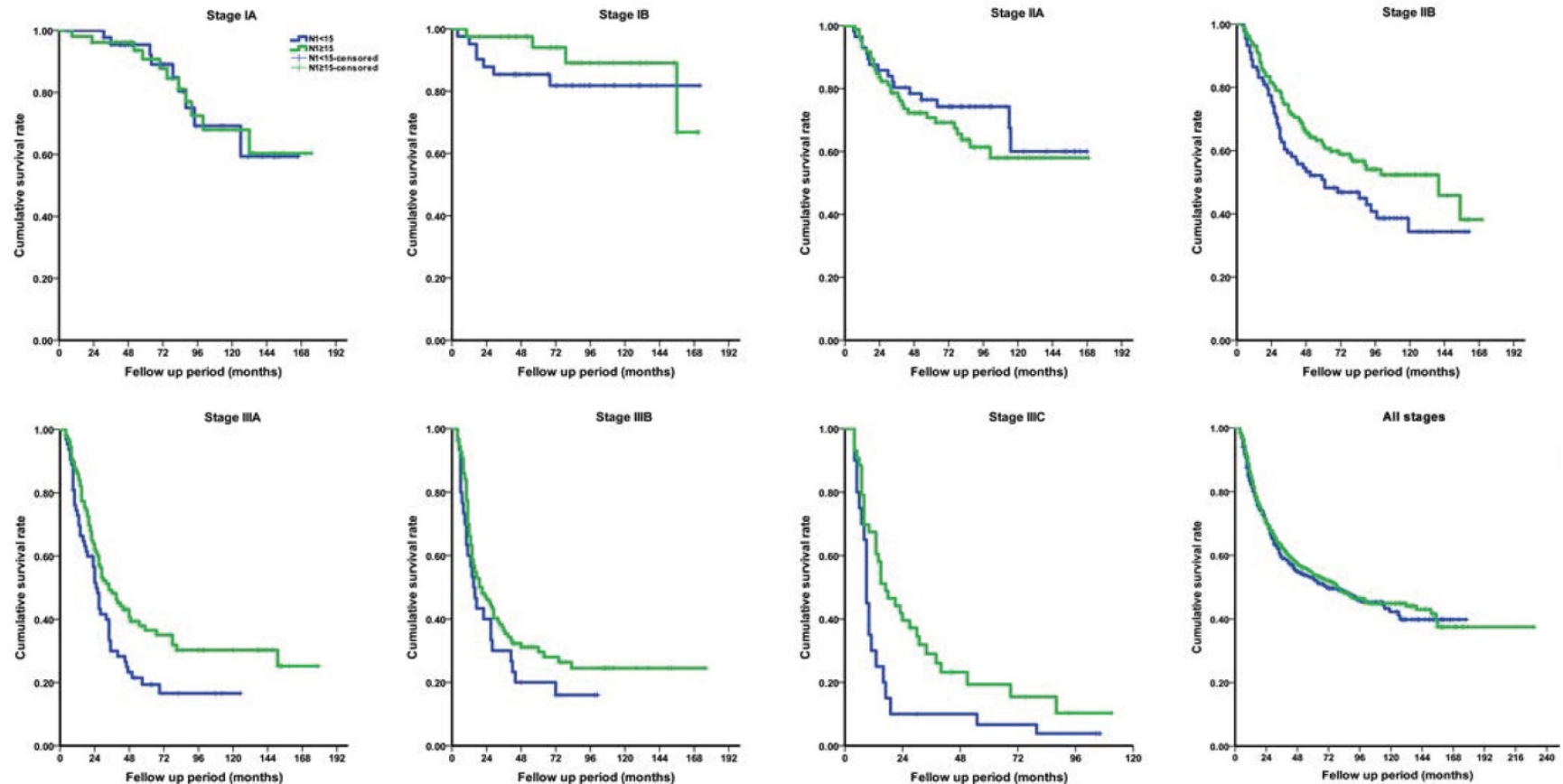

Figure 4. Comparison of long-term overall survival rate between study group $(\mathrm{NI} \geq 15)$ and control group $(\mathrm{N} 1<15)$ according to pathological stage. (A) stage IA; (B) stage IB; (C) stage IIA; (D) stage IIB; (E) stage IIIA; $(F)$ stage IIIB; (G) stage IIIC; (H) all stages.

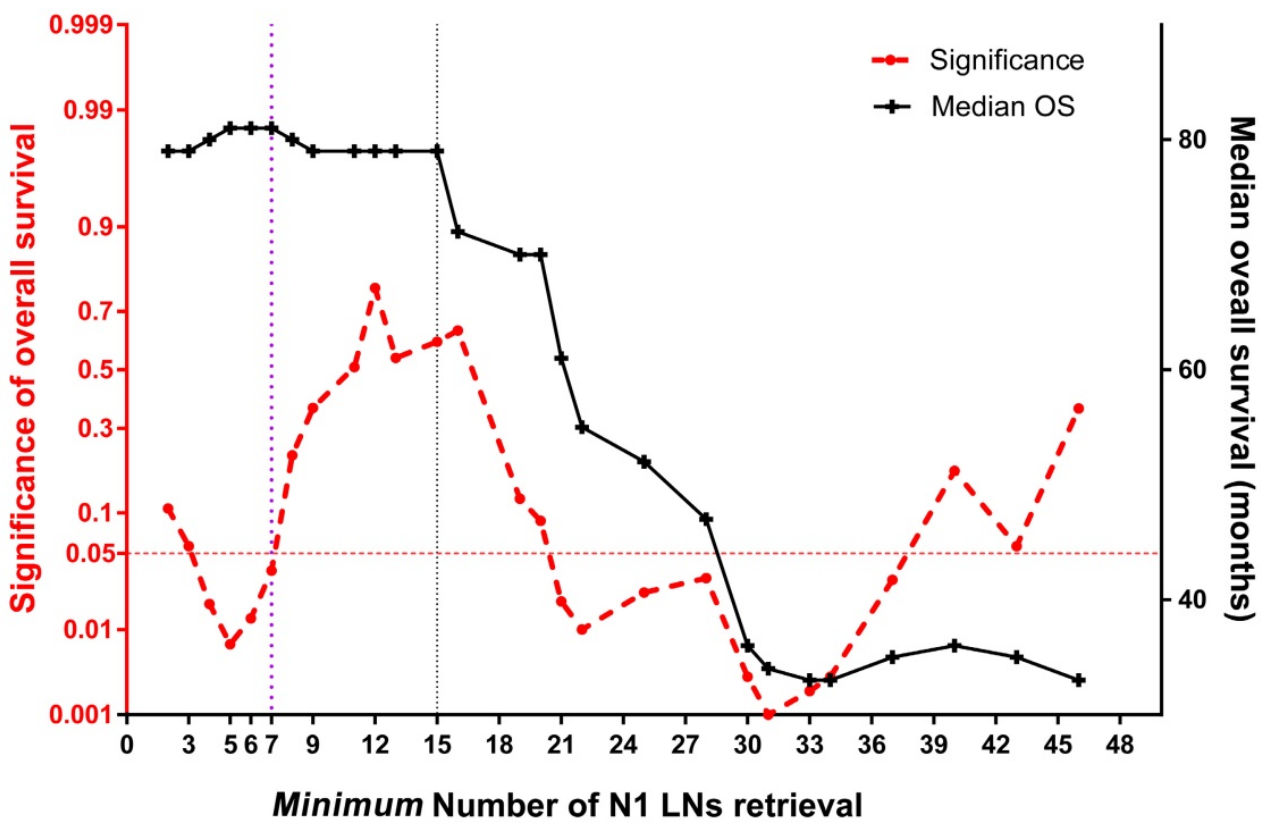

Figure 5. The joinpoint trend analysis for median survival at each count of perigastric LNs retrieval. The red dotted line below 0.05 at left $Y$-axis indicates survival significance in the current cohort; the black line indicates the median overall survival (right $Y$-axis), which slopes down at 7 and $15 X$-axis, respectively. 
It is well recognized that anatomical location of metastatic LNs is an indispensable prognostic factor for GC patients [27, 28]. The presence of metastases in regional LNs is related to perigastric tumor deposits, advanced tumor size and invasion depth [29, 30]. Several studies have suggested that the metastatic number of $\mathrm{N} 1$ nodes can be regarded as a simple parameter for predicting long-term survival [31], with more than three positive nodes considered as poor prognosis [19, 20, 32]. To our knowledge, the number of $\mathrm{N} 1$ nodes retrieval is more convenient than that of metastatic N1 nodes retrieval to evaluate nodal status and quality of lymphadenectomy, especially in advance of available pathological $\mathrm{N}$ staging. Perigastric LNs may act as a line of defense to prevent metastases to extraperigastric LNs, with a limited bypassing nodal metastasis [33]. Our study, which focused on the amount of perigastric LNs retrieval rather than metastatic involvement in regional LNs, have confirmed their strong correlations and provided a clue that incremental number of harvested N1 nodes remains survival benefits until the count of 15 nodes.

In this study, additional joinpoint trend analysis using each count of perigastric LN retrieval was performed to confirm an optimal harvested number for a survival significance. Although two joinpoints were revealed, only at least seven nodes retrieved in N1 stations was associated with survival significance. Unfortunately, subdivided groups separated by seven N1 nodes were distinctly unbalanced, with only 70 $(7.0 \%)$ patients included in the control group. As a result, the statistical power of survival analysis under such cut-off level of nodal retrieval would be depressed in some way. In clinical practice, harvest of at least $15 \mathrm{~N} 1$ nodes would rather be suggested since the median survival time started to decline after such joinpoint (Figure 5). To our best knowledge, this is the first time that a detailed quantitative comparison of perigastric LNs is made for long-term survival outcome of GC patients.

This study had several limitations, which should be mentioned. First, this single-center retrospective study, which included subjects within a huge range of time scales, would have heterogeneous quality control of lymphadenectomy or pathological technique. Therefore, tumor stage migration, especially for $\mathrm{N}$ category, might be unavoidable in certain times. Second, the impact of adjuvant chemotherapy on LNs retrieval or overall survival was not evaluated for this cohort. Such treatment may conceal survival significance of specific number of nodal retrieval in the perigastric region. Last, as a result from a large distributed study period, the applied tumor staging and surgical strategies were hardly unified in comparison with a prospective study. It is believed that more available data from well-designed studies with large-scale would further confirm a proper number of perigastric LNs retrieval.

\section{Conclusion}

Lymphadenectomy with adequate harvest of perigastric lymph nodes is essential for resectable gastric cancer. Our work for the first time explored the role of perigastric nodal retrieval in predicting long-term outcomes. It suggests that at least 7-15 LNs should be retrieved or dissected from perigastric stations to achieve a better survival benefit. As a guide to clinical practice, en bloc resection combined with high-quality LN harvest and precise station mark should be more emphasized to get the best oncologic outcomes.

\section{Acknowledgements}

We acknowledge financial support from National Natural Science Foundation of China (No. 81401307) and Medical science and technology research funding project in Guangdong province (No. A2015112 \& NO.2016A0103038). We gratitude Miss. Donglian Chen and Miss. Qiaojie Lin for valuable helps in database management and follow-up visits.

\section{Competing Interests}

The authors have declared that no competing interest exists.

\section{References}

1. Karpeh MS, Leon L, Klimstra D, Brennan MF. Lymph node staging in gastric cancer: is location more important than Number? An analysis of 1,038 patients. Ann Surg. 2000; 232: 362-371.

2. Mocellin S, McCulloch P, Kazi H, Gama-Rodrigues JJ, Yuan Y, Nitti D. Extent of lymph node dissection for adenocarcinoma of the stomach. Cochrane Database Syst Rev. 2015: CD001964.

3. Kim YI. Is retrieval of at least 15 lymph nodes sufficient recommendation in early gastric cancer? Ann Surg Treat Res. 2014; 87: 180-184.

4. Japanese Gastric Cancer A. Japanese gastric cancer treatment guidelines 2010 (ver. 3). Gastric Cancer. 2011; 14: 113-123.

5. Doglietto GB, Rosa F, Bossola M, Pacelli F. Lymphadenectomy for gastric cancer: still a matter of debate? Ann Ital Chir. 2012; 83: 199-207.

6. Bouvier AM, Haas O, Piard F, Roignot P, Bonithon-Kopp C, Faivre J. How many nodes must be examined to accurately stage gastric carcinomas? Results from a population based study. Cancer. 2002; 94: 2862-2866.

7. Baiocchi GL, Tiberio GA, Minicozzi AM, Morgagni P, Marrelli D, Bruno L, et al. A multicentric Western analysis of prognostic factors in advanced, node-negative gastric cancer patients. Ann Surg. 2010; 252: 70-73.

8. Smith DD, Schwarz RR, Schwarz RE. Impact of total lymph node count on staging and survival after gastrectomy for gastric cancer: data from a large US-population database. J Clin Oncol. 2005; 23: 7114-7124.

9. Washington K. 7th edition of the AJCC cancer staging manual: stomach. Ann Surg Oncol. 2010; 17: 3077-3079.

10. Japanese Gastric Cancer A. Japanese gastric cancer treatment guidelines 2014 (ver. 4). Gastric Cancer. 2017; 20: 1-19.

11. Song W, Yuan Y, Wang L, He W, Zhang X, Chen C, et al. The prognostic value of lymph nodes dissection number on survival of patients with lymph node-negative gastric cancer. Gastroenterol Res Pract. 2014; 2014: 603194.

12. Kajitani T. The general rules for the gastric cancer study in surgery and pathology. Part I. Clinical classification. Jpn J Surg. 1981; 11: 127-139.

13. Woo Y, Goldner B, Ituarte $\mathrm{P}$, Lee B, Melstrom L, Son $\mathrm{T}$, et al. Lymphadenectomy with Optimum of 29 Lymph Nodes Retrieved Associated with Improved Survival in Advanced Gastric Cancer: A 25,000-Patient International Database Study. J Am Coll Surg. 2017; 224: 546-555.

14. Hu B, El Hajj N, Sittler S, Lammert N, Barnes R, Meloni-Ehrig A. Gastric cancer: Classification, histology and application of molecular pathology. J Gastrointest Oncol. 2012; 3: 251-261. 
15. Jiang L, Yao Z, Zhang Y, Hu J, Zhao D, Zhai H, et al. Comparison of lymph node number and prognosis in gastric cancer patients with perigastric lymph nodes retrieved by surgeons and pathologists. Chin J Cancer Res. 2016; 28 : 511-518.

16. Shi RL, Chen Q, Ding JB, Yang Z, Pan G, Jiang D, et al. Increased number of negative lymph nodes is associated with improved survival outcome in node positive gastric cancer following radical gastrectomy. Oncotarget. 2016; 7: 35084-35091.

17. Zheng G, Feng F, Guo M, Xu G, Liu S, Liu Z, et al. Harvest of at Least 23 Lymph Nodes is Indispensable for Stage N3 Gastric Cancer Patients. Ann Surg Oncol. 2017; 24: 998-1002.

18. Deng J, Zhang R, Pan Y, Wang B, Wu L, Jiao X, et al. Comparison of the staging of regional lymph nodes using the sixth and seventh editions of the tumor-node-metastasis (TNM) classification system for the evaluation of overall survival in gastric cancer patients: findings of a case-control analysis involving a single institution in China. Surgery. 2014; 156: 64-74.

19. Yu W, Kim HS, Choi GS, Suh IS. Perigastric lymph nodes with metastasis in gastric cancer. Hepatogastroenterology. 1999; 46: 2658-2661.

20. Chikara K, Hiroshi S, Masato N, Goro M, Yuichi O, Hidetaka O, et al. Association of the number of metastatic perigastric lymph nodes with long-term survival in gastric cancer. Hepatogastroenterology. 2005; 52: 277-280.

21. Okajima W, Komatsu S, Ichikawa D, Kosuga T, Kubota T, Okamoto K, et al. Prognostic impact of the number of retrieved lymph nodes in patients with gastric cancer. J Gastroenterol Hepatol. 2016; 31: 1566-1571.

22. Degiuli M, Sasako M, Ponti A, Vendrame A, Tomatis M, Mazza C, et al. Randomized clinical trial comparing survival after D1 or D2 gastrectomy for gastric cancer. Br J Surg. 2014; 101: 23-31.

23. Hu Y, Huang C, Sun Y, Su X, Cao H, Hu J, et al. Morbidity and Mortality of Laparoscopic Versus Open D2 Distal Gastrectomy for Advanced Gastric Cancer: A Randomized Controlled Trial. J Clin Oncol. 2016; 34: 1350-1357.

24. Inaki N, Etoh T, Ohyama T, Uchiyama K, Katada N, Koeda K, et al. A Multi-institutional, Prospective, Phase II Feasibility Study of Laparoscopy-Assisted Distal Gastrectomy with D2 Lymph Node Dissection for Locally Advanced Gastric Cancer (JLSSG0901). World J Surg. 2015; 39: 2734-2741.

25. Galizia G, Lieto E, De Vita F, Castellano P, Ferraraccio F, Zamboli A, et al. Modified versus standard D2 lymphadenectomy in total gastrectomy for nonjunctional gastric carcinoma with lymph node metastasis. Surgery. 2015; 157: 285-296.

26. Kim W, Kim HH, Han SU, Kim MC, Hyung WJ, Ryu SW, et al. Decreased Morbidity of Laparoscopic Distal Gastrectomy Compared With Open Distal Gastrectomy for Stage I Gastric Cancer: Short-term Outcomes From a Multicenter Randomized Controlled Trial (KLASS-01). Ann Surg. 2016; 263: 28-35.

27. de Manzoni G, Verlato G, di Leo A, Guglielmi A, Laterza E, Ricci F, et al. Perigastric lymph node metastases in gastric cancer: comparison of different staging systems. Gastric Cancer. 1999; 2: 201-205.

28. Zhao B, Zhang J, Zhang J, Chen X, Chen J, Wang Z, et al. Anatomical location of metastatic lymph nodes: an indispensable prognostic factor for gastric cancer patients who underwent curative resection. Scand J Gastroenterol. 2018; 53: 185-192.

29. Cambruzzi E, Azeredo AM, Kronhart A, Foltz KM, Zettler CG, Pegas KL. The presence of metastases in regional lymph nodes is associated with tumor size and depth of invasion in sporadic gastric adenocarcinoma. Arq Bras Cir Dig. 2014; 27: 18-21.

30. Anup S, Lu J, Zheng CH, Li P, Xie JW, Wang JB, et al. Prognostic significance of perigastric tumor deposits in patients with primary gastric cancer. BMC Surg. 2017; 17: 84 .

31. Deng JY, Liang H, Sun D, Pan Y, Liu Y, Wang DC. Extended lymphadenectomy improvement of overall survival of gastric cancer patients with perigastric node metastasis. Langenbecks Archives of Surgery. 2011;396: 615-623.

32. Kim TH, Han SU, Cho YK, Kim MW. Perigastric lymph node status can be a simple prognostic parameter in patients with gastric cancer. Hepatogastroenterology. 2000; 47: 1475-1478.

33. Ichikura T, Furuya Y, Tomimatsu S, Okusa Y, Ogawa T, Mukoda K, et al. Relationship between nodal stage and the number of dissected perigastric nodes in gastric cancer. Surgery today. 1998; 28: 879-883. 